\title{
Performance Prediction with Skeletons
}

\author{
Sukhdeep Sodhi Jaspal Subhlok Qiang Xu
}

\begin{abstract}
The performance skeleton of an application is a short running program whose performance in any scenario reflects the performance of the application it represents. Specifically, the execution time of the performance skeleton is a small fixed fraction of the execution time of the corresponding application in any execution environment. Such a skeleton can be employed to quickly estimate the performance of a large application under existing network and node sharing. This paper presents a framework for automatic construction of performance skeletons of a specified execution time and evaluates their use in performance prediction with CPU and network sharing. The approach is based on capturing the execution behavior of an application and automatically generating a synthetic skeleton program that reflects that execution behavior. The paper demonstrates that performance skeletons running for a few seconds can predict the application execution time fairly accurately. Relationship of skeleton execution time, application characteristics, and nature of resource sharing, to accuracy of skeleton based performance prediction, is analyzed in detail. The goal of this research is accurate performance estimation in heterogeneous and shared computational grids.
\end{abstract}

\section{Introduction}

Computational grids are emerging as the vehicle for future high performance scientific and commercial computing. Execution environments for grids have to address allocation of resources to applications, and that is driven by the expected performance of an application on different parts of a grid. Estimation of application performance has an important role to play in grid computing, and the problem is much more complex for a shared heterogeneous computation environment than for conventional high performance computing platforms.

A large body of research addresses resource selection for grid applications, and several projects are discussed in section 2 . The state of the art can be broadly summarized as consisting of the following steps:

1. System characterization: Measure and predict the status and availability of system resources such as CPU and network capacities. 
2. Application characterization: Develop a model that captures the dependence of an application's performance on availability of resources.

3. Mapping and scheduling: Select the best nodes to execute the application based on available system status and application characteristics.

We argue that this state of the art has the following inherent limitations that motivate a different paradigm:

- Maintaining accurate current system status information is inherently expensive. In order to have recent CPU and network information whenever a resource assignment decision has to be made, available system resources have to be monitored continuously and status information has to be broadcast frequently. For network properties, measurements themselves consume bandwidth and the complexity increases quadratically with the size of the available computing grid. High speed backbone network links are particularly challenging since it is not desirable to consume a critical shared resource for measurements.

- Estimating application performance based on system status is inherently error prone. Measurement tools provide resource availability and utilization information such as CPU load factor and unused bandwidth on various components of a grid. On the other hand, the key information of interest for resource management is how a particular application will perform on a set of resources under the current system status. Predicting the performance of application tasks from system status information is very difficult. The following examples underline the complexity:

- The amount of CPU time that a process is likely to get on a computation node cannot be determined even when the load average on the node is known since it partly depends on the synchronization structure of the parallel and distributed applications in the system.

- The expected duration of a bulk transfer cannot be estimated accurately even when accurate point to point unused bandwidth information is available since it depends on the transport protocols used by the application and other traffic on the network.

Finally even if the performance on individual node computations and data transfers can be determined, estimating collective communication and overall application performance is still challenging as it depends on the nature of sharing in the network and the application structure.

The conclusion is that it is virtually impossible to estimate application performance from network status in many scenarios. This has motivated us to follow a different approach to estimating performance in shared heterogeneous grid environments which is based on the following claim: 
The most effective and efficient way to estimate the performance of an application under the existing status of grid resources is brief monitored execution of code that mimics the application.

We refer to such code as the performance skeleton of the application. More formally, a performance skeleton is a synthetically generated short running program whose execution time always reflects the performance of the application it represents. Hence, simply executing the performance skeleton in a shared execution environment provides an estimate of the application performance in that environment. The resource selection for an application is then addressed as follows. A group of candidate node sets is identified for execution (using existing approximate methods) and the final choice is made by comparing the execution time of the application skeleton on each node set.

The central contribution of this paper is a framework for automatic construction of accurate performance skeletons for distributed applications and evaluation of the capability of automatically generated skeletons to predict performance efficiently and accurately.

While we have used resource selection in shared grid environments for motivating this research, it is important to point out that this approach to performance prediction has broad applicability. Another example is prediction of the performance of important applications on a future architecture under simulation. Since execution under simulation is multiple orders of magnitude slower than real execution, this skeleton based approach can be particularly appropriate. The real application does not have to be simulated at all as the skeleton can be built on existing machines.

The basic philosophy in construction of a performance skeleton can be stated as follows. If the skeleton executes operations that are representative of application execution, the performance of the skeleton and the application will change similarly in response to changes in the execution environment. Hence, a performance skeleton must capture the execution behavior of the application in terms of synchronization and message exchange patterns, CPU usage patterns, and memory access patterns, yet execute for a very short time. Our approach is to measure the application performance behavior during execution, summarize it by identifying repeating phases, and then reproduce it as a synthetic skeleton program.

This paper is organized as follows. Section 2 discusses related research in resource management and performance prediction in grid environments. Section 3 explains the concept of a performance skeleton and section 4 describes the procedure to construct performance skeletons. Section 5 presents results that illustrate the accuracy of skeleton based performance prediction. Section 6 discusses the current limitations and possible extensions of this approach and section 7 contains conclusions. 


\section{Related work}

The research community clearly recognizes the importance of performance estimation in grid environments and substantial research effort has been invested in the measurement, modeling, and prediction of various system resources. Measurement and prediction of CPU availability has been studied in [11,37]. Measurement and modeling of network bandwidth and latency is a very active area of research $[7,14,21,30]$. NWS (Network Weather Service) [38] and REMOS (Resource Monitoring System) [17] are two systems that have been specifically designed for measurement of available CPU and network resources in grid environments. NWS, in particular, is in widespread use as a CPU and bandwidth monitoring and prediction tool.

Systems for resource management and scheduling for problem solving on grid environments include Netsolve [8], Nimrod/G [6], Gallop [36], AppLeS [4] and Condor [16, 22]. These systems rely on measured and predicted availability of CPU, bandwidth and other resources to make resource allocation and management decisions where applicable. AppLeS [4] pioneered application level scheduling, where resource selection is performed by agents associated with an application based on available resource information, rather than by a central resource manager. A number of algorithms and frameworks have been proposed for resource selection in networked environments based on system status information, some examples being $[4,31]$. Some of the recent research has emphasized the importance of application properties in resource allocation and addresses resource selection based on mapping application properties to the system status [5, 9, 23, 28, 33, 35].

We specifically discuss other projects that focus on capturing application behavior and application performance. FAST [10] is a tool that performs abstract simulations while completely avoiding execution of computation code. Reed et. al. [18] generate compact application signatures that summarize time varying application behavior while still retaining the compactness of statistical summaries. They use a curve-fitting approach that reduces event-tracing overheads for online performance monitoring and tuning but still retains many of the advantages of event-tracing. Snavely et.al. [27, 28] create application and machine signatures to simulate application behavior across different system or processor architectures, and yield performance predictions by convolving application signatures with a machine signature. Our approach is driven by many of the ideas and concepts developed in these projects. However, we have a very different goal, which is to develop an executable skeleton program, rather than a performance model.

The skeleton construction procedure relies on repetitive application behavior to be effective. There is abundant evidence that this is the case for practical applications. Duesterwald et.al. [12] explore metrics derived from hardware counters to characterize program behavior and its variability. They discover that phase/periodicity behavior is shared across different metrics and use it for kernel-level resource aware scheduling. Sherwood et.al. [24, 25] also exploit periodic application behavior by building basic block vectors. They identify portions of the program that are representative of an application for the purpose of archi- 
tectural simulations.

Yang et.al. [15] show that performance translation and prediction derived from very short partial executions can yield high accuracy at a low cost. The reason is that most parallel codes are iterative and behave in a predictable manner after a minimal startup period. Nakazaw et.al. [20] propose an execution modelMHETA, which takes into account computation, communication, and I/O costs of iterative scientific applications. This model automatically uses extracted information from a single iteration to predict the execution time of the remaining iterations. In contrast, a performance skeleton is based on a full analysis of application execution. This allows accurate performance estimation across a wider class of applications including those with multiple diverse phases. Also, a performance skeleton is a stand-alone program that can yield a performance estimate without even invoking the full application in a new environment.

\section{Performance skeletons}

A performance skeleton is defined as a program whose execution time is directly related to the execution time of the application it represents; if the execution time of a skeleton is 1/1000th of the application execution time on a dedicated cluster, then this relationship should hold in any execution environment, even when nodes and links are shared with other applications. This definition is idealistic, and in practice, the goal is to build a skeleton that conforms to these conditions as closely as possible. The skeleton should also be as short-running as possible as skeleton execution is an overhead. We would like to point out that skeleton execution is very different from executing the application for a short time. The skeleton should capture the total execution of an application in a short time while the beginning part of an application is typically not representative of the entire application execution.

For the performance behavior of a skeleton to be similar to that of an application, the execution and resource usage patterns of the skeleton must be similar to the dominant corresponding patterns of the application. We have the following specific criteria:

1. CPU activity: The processing done by the CPU and CPU busy/idle phase pattern should be similar for the application and the skeleton.

2. Memory activity: The memory access pattern in the skeleton should be representative of the application. This is particularly important to get similar cache performance on nodes with different memory hierarchies.

3. I/O activity: The I/O pattern in the skeleton should be representative of the application.

4. Communication and synchronization: The data exchange patterns among processes should be similar for the application and the skeleton to preserve the communication and synchronization performance. 
The sizes, types, frequencies and patterns of network message exchanges should be similar.

5. Application phase transitions: An application transitions between different phases of execution at multiple levels of granularity. The sequence of these phases, as well as the CPU, memory and communication activities in each phase, should be reflected in the skeleton.

Our long term project goal is to generate skeletons conforming to the above constructive definition but this paper is limited to performance skeletons which mimic the communication sequences and coarse computation behavior of the application. Such skeletons are sufficient for predicting performance of compute and communication bound applications under resource sharing. Reproduction of memory accesses and fine-grain instruction level computation behavior is critical for performance estimation across different processor and memory architectures, but not essential for simple CPU and network sharing scenarios. We discuss our efforts in reproducing memory behavior for performance prediction in [34].

\section{Automatic construction of skeletons}

This research has developed a framework for automatic construction of performance skeletons and implemented it for message passing MPI programs. We outline the procedure in this section. The main steps are as follows:

1. Record application's execution trace: The application is executed on a controlled testbed and its execution activity, specifically CPU usage and message exchanges, is recorded. This is the execution trace.

2. Compress execution trace into an execution signature: The repeated patterns in the recorded execution trace are identified and used to generate a compact representation of the trace by introducing a "loop structure". The new compact representation is the execution signature.

3. Generate performance skeleton program from the execution signature: The application execution signature is converted to a computer program which generates execution activity that is similar to the recorded execution signature, but with the execution time scaled down by a given factor K. This is the performance skeleton.

The skeleton construction procedure is illustrated in Figure 1. This procedure does not involve source code analysis, modification or instrumentation and hence has broad applicability. Skeleton construction is driven by the desired ratio between the execution time of the application and the corresponding performance skeleton, which we call the scaling factor. We now discuss each of these steps. Additional details are available in [29]. 


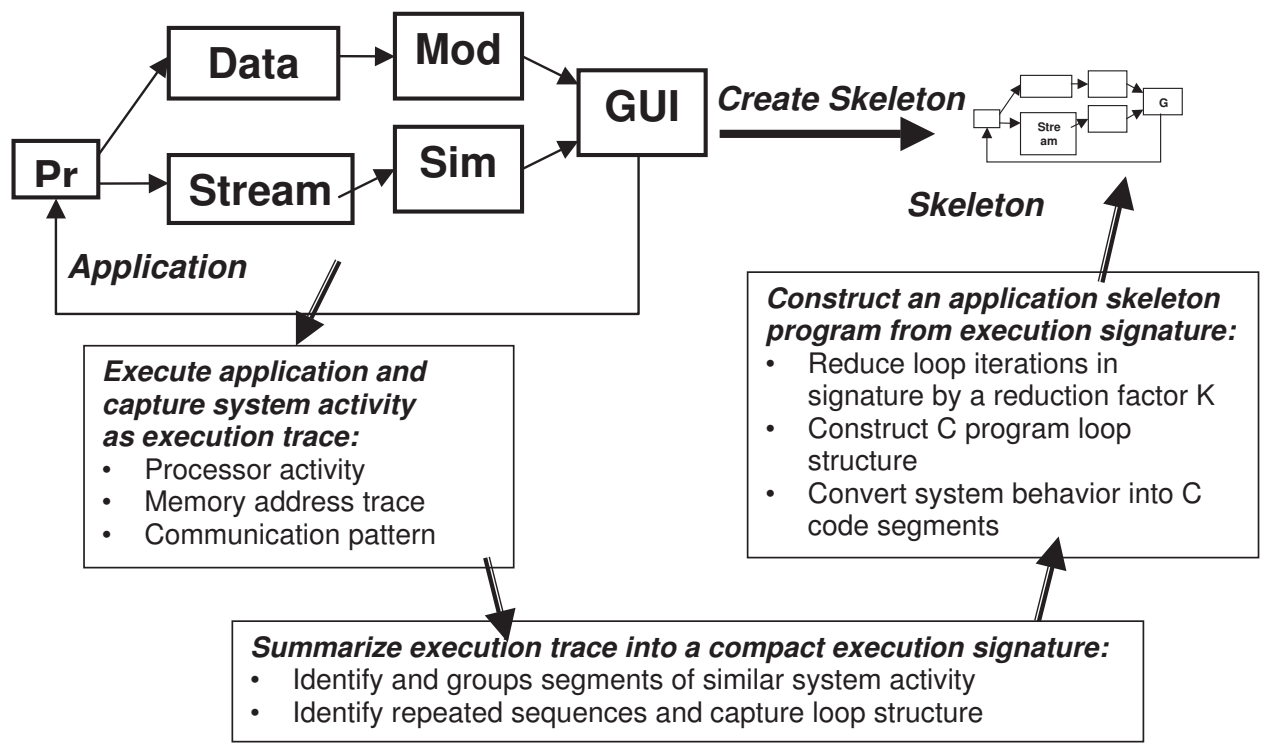

Figure 1: Construction of application performance skeletons.

\subsection{Recording of execution trace}

To generate an execution trace, the MPI application is linked with a profiling library by employing the standard PMPI interface [19] to intercept all MPI calls. When an MPI call is executed in the original application, the control is transferred to the profiling library. The library records the call parameters and the time stamp, and then invokes the original MPI routine for actual communication. When the control returns to the profiling library, another time stamp representing the end of the communication operation is recorded and the control is returned to the original calling routine.

After linking to enable profiling, the application is executed on a dedicated testbed cluster without any competing jobs or network traffic. The profiling library records information for each application process in a separate trace file. Each MPI library call, along with the parameters passed to it and its start time and end time, are recorded. Timing measurement is done to microsecond granularity with Linux gettimeofday system call [1]. Time for computation operations is recorded as the time spent between the end of one MPI operation and the start of the next MPI operation. Generation of the trace file requires no modification of the application source code. We verified that the execution time overhead of trace generation is negligible, typically well under $1 \%$ of the execution time.

\subsection{Compression of execution trace to execution signature}

The application execution trace is a long record of message exchanges and interleaved compute operations of varying duration. The bulk of an application's execution time is typically spent in repeating loops as 
application execution activity tends to be cyclic. The goal of this step is to identify cyclic behavior in the execution trace to generate a compact execution signature. Segments of execution trace denoting similar activity qualify for repeating behavior; exactly identical activity is not required. This process consists of clustering similar execution events in the trace followed by the conversion of the repeated operation sequences into a loop structure.

\section{Clustering similar execution events:}

The objective of this stage is to replace the execution trace by a string of symbols where substantially similar execution events are placed in the same cluster and assigned the same symbol.

As an example, suppose we encounter the following two operations in a trace:

MPI_Send(Node 3, 2000 bytes), and MPI_Send(Node 3, 1800 bytes)

If both these events occur only once, they are both replaced by the following operation:

\section{MPI_Send(Node 3, 1900 bytes)}

Clustering such similar events helps in generating a more compact representation. Events that are grouped together are execution phases of approximately equal duration or message calls with similar parameters. Our approach treats different MPI primitives and blocking and non-blocking calls as distinct events, thus ensuring that they are never grouped together. Further our approach ensures that point-to-point calls between different pairs of nodes and collective calls with different root nodes are never grouped together. We identify the non blocking calls and associated MPI_Wait() to determine the corresponding overlapped region. This helps develop a faithful representation of the application's communication structure.

Formally, we have developed a measure for dissimilarity of events in $\mathrm{N}$-dimensional space based on [13], with one dimension for each parameter of an execution event. The extent of clustering is controlled by a similarity threshold which can be assigned a value between 0 and 1 . A lower similarity threshold represents more strict rules for clustering but will lead to less compression, while a higher similarity threshold implies more relaxed rules for clustering and more compression. A similarity threshold of 0 implies that only identical events are clustered together.

This stage converts the trace log into a string of symbols such as:

$$
\alpha \beta \beta \gamma \beta \beta \gamma \beta \beta \gamma \kappa \alpha \alpha
$$

where each occurrence of a symbol represents an execution event with different occurrences of the same symbol referring to functionally identical execution events.

To summarize, clustering of similar events and representing them by an "average event" implies some loss of information but leads to significant compression, and subsequently, smaller skeletons. This 
tradeoff can be managed with the similarity threshold parameter as discussed.

\section{Identification of cycles:}

The objective of this step is to identify and capture repeated execution behavior as loops to construct the final execution signature. Since the previous step converts the execution trace into a sequence of frequently repeating symbols, the problem of identifying repeating application execution behavior is now represented as the problem of finding repeating sub-strings within a string. As an example, the following string:

$$
\alpha \beta \beta \gamma \beta \beta \gamma \beta \beta \gamma \kappa \alpha \alpha
$$

should be replaced by:

$$
\alpha\left[(\beta)^{2} \gamma\right]^{3} \kappa[\alpha]^{2}
$$

The procedure consists of recursively identifying the repeating sub-strings, starting with the largest matches and working down to sub-string matches of a single symbol. The repeating sub-strings are then organized as recursive loop nests with sub-strings of symbols as loop bodies and the number of repetitions as the number of loop iterations. This algorithm is outlined in Table 1. A straightforward analysis shows that the complexity of the compression algorithm is $O\left(N^{3}\right)$ for a string of $N$ symbols.

An important parameter in the procedure for the construction of an execution signature is the similarity threshold, which determines if two similar events can be considered identical for the purpose of compression. We now address how a given value of similarity threshold translates to specific rules for compression and then discuss how the value of similarity threshold is determined. For message passing operations, the value of the similarity threshold linearly relates to the maximum difference in message sizes allowed for communication operations to be combined into a cluster. The above compression procedure is applied across communication operations without regard to interleaving computations. When two sequences of communication events with interspersed computation events are to be combined, an average value of execution time for the corresponding computation events in the sequence is used to build the compressed sequence. This approach represents maximum flexibility in combining computation events and was found to be effective in our experience.

An iterative process outlined in Table 2 is employed to determine the optimal value of the similarity threshold based on the desired compression ratio $Q$ between the length of the execution trace and the length of the compressed execution signature. Initially the similarity threshold is set to 0 and the clustering and compression procedure is applied. If the degree of compression is less than the desired ratio Q, the similarity threshold is increased gradually until the desired compression of Q (or higher) is achieved. Now, the question is how should $\mathrm{Q}$ be determined ? Based on our experience, we have used $\mathrm{Q}=\mathrm{K} / 2$ where $\mathrm{K}$ is the scaling factor between the application execution time and the desired skeleton execution time. It is desirable to have an upper bound on similarity threshold so that very different execution events are not combined. In practice, 
Table 1: Algorithm to compress strings by finding repeating sub-strings

INPUT: S; a string of symbols.

OUTPUT: A String table of tuples $\left(S_{0}, c_{0}\right),\left(S_{1}, c_{1}\right) \ldots\left(S_{k}, c_{k}\right) . S_{0}$ represents the compressed string.

Each string $S_{i}$ is a sequence of symbols and other strings.

Each $c_{i}$ is the number of repetitions of the corresponding string.

1. Initialization: $i=1 ; S_{0}=S$;

2. $w=$ length $\left(S_{0}\right) / 2$;

3. If $S_{0}$ contains a repeating substring of length $w$ then go to step 4. Else go to step 5 .

4. Let $S_{i}$ be one repeating substring of size $w$ that repeats $c_{i}$ times.

Add $\left(S_{i}, c_{i}\right)$ to the string table.

Replace the sequence of repeated strings in $S_{0}$ with symbol $S_{i}$.

Increment $i=i+1$;

Go to step 2

5. Decrement $w=w-1$; If $w>0$ go to step 3 Else add $S_{0}$ to the string table and Stop.

this may not be a significant issue. The similarity threshold that was required across the NAS benchmarks for meaningful execution signatures was frequently 0 , and always less than .2. The implication is that only combining closely similar events may be sufficient for skeleton generation in practice.

\subsection{Generation of performance skeleton program from execution signature}

The previous stage gave us the execution signature which is a compressed record of the complete execution of the application. The execution signature compresses execution information by using a loop structure with loop bodies representing repeating execution behavior. Our goal in this step is to create a short running program in a programming language like $\mathrm{C} / \mathrm{C}++$ which reproduces the scaled down dominant execution behavior represented by the execution signature. The specific goal is to take the application's execution signature and the desired scaling factor $\mathrm{K}$ as inputs, and generate an appropriate performance skeleton. The skeleton construction procedure is outlined as follows:

1. The numbers of loop iterations in the application signature are reduced by a factor K. Loop iterations that form the remainder in this division process are unrolled and become a component of the unreduced part of the signature. 
Table 2: Algorithm used to generate execution signature from the execution trace of an application

INPUT: Execution trace of an application from a single node.

OUTPUT: Execution signature of the application represented as a compressed string $S_{0}$.

\section{INITIALIZE}

SimilarityThreshold $=$ initial value; (usually 0 )

$K=$ Application execution time / Desired skeleton execution time;

$M=$ Null; (Table mapping processed MPI operations to symbols)

1. Replace each MPI operation in the trace by a symbol. Represent similar operations by the same symbol.

2. For each operation $O p$ in trace

Find $\mathrm{MinOp}$ (operation in $\mathrm{M}$ which is most similar to $O p$ ).

If (Dissimilarity $(O p, M i n O p)<$ SimilarityThreshold)

Then Represent $O p$ in $\mathrm{S}$ by corresponding symbol for MinOp.

Else Represent $O p$ by a new symbol in $\mathrm{S}$ and add this information to $M$.

3. Compress resultant trace $S$ using the algorithm in Table 1 yielding $S_{0}$.

Compression Ratio $Q=\operatorname{length}(S) /$ length $\left(S_{0}\right)$;

4. If $(Q<K / 2$ and SimilarityThreshold $<$ max allowed $)$

Then increment SimilarityThreshold. Goto Step 1.

Else Return $\left(S_{0}\right)$. Stop.

This step typically generates the dominant part of the execution skeleton. If the bulk of the execution trace is represented in the execution signature as loops with a large number of iterations, the remaining steps, which handle the non-loop part of the execution signature, only make a marginal difference and can be omitted

2. Groups of K occurrences of identical execution operations anywhere in the unreduced part of the skeleton are identified and replaced by a single occurrence.

3. All remaining unreduced operations are scaled down by a factor $\mathrm{K}$ by adjusting their parameters. For compute operations, the duration of execution is reduced by a factor K. For communication operations, the number of bytes exchanged is reduced by a factor $\mathrm{K}$. 
4. This modified application signature is converted to synthetic $\mathrm{C}$ code by generating corresponding synthetic loops, MPI calls, and compute operations.

One weakness of this approach is that scaling down a communication operation by reducing the number of bytes exchanged is not accurate. Execution time of the reduced operation would typically be higher than expected because communication operations have two time components; latency, which is fixed for all message sizes, and message transfer time, which can be scaled down linearly. By reducing the number of bytes exchanged we only reduce the message transfer time, leaving the latency component intact. A more accurate scaling cannot be achieved without making some assumptions about the execution environments. Also, we point out that this kind of reduction is a "last resort" that is employed only for iterations that remain after division by $\mathrm{K}$ and for operations not in loops. In practice, the impact on overall performance estimation is expected to be minimal for most applications.

\subsection{Skeleton construction usage and example}

The skeleton construction framework is fully automated. In order to build a performance skeleton of an application, a user takes the following actions. The first step is to link the application with a provided profiling library and execute in a sample cluster execution environment. This results in the generation of a set of trace files, one per executing thread. Next, the main skeleton generation program is executed with the trace files as input and the scaling factor between the desired skeleton and application execution times as a parameter. A performance skeleton is generated as the output. This performance skeleton is a portable C/MPI program that can be executed in different execution environments.

We selected the class S BT NAS benchmark as a realistic example program to illustrate the construction of a performance skeleton from an execution trace. The original execution trace is shown in Table 3. The trace was recorded on Node 0 . The trace consists of 2278 MPI calls, with computation sections between them. Table 3 lists samples from the beginning and end of the trace. This trace only lists the communication calls, which are always interleaved with computation sections that are omitted for brevity. The general format of the communication calls in the execution trace is as follows:

MPICallName\#Parameter_1\#Parameter_2...\#Parameter_n\#StartTime\#EndTime where the number and meaning of parameters depends on the call.

For example the first line in the trace in Table 3 is as follows:

2\#1\#3220724724\#1\#28\#0\#134\#0\#939220509\#939220509

The fields, from left to right, and their values in this trace entry are as follows:

Type of call: (2 representing MPI_BCast)

Occurrence number of that call in the trace (1) 
Table 3: Execution Trace

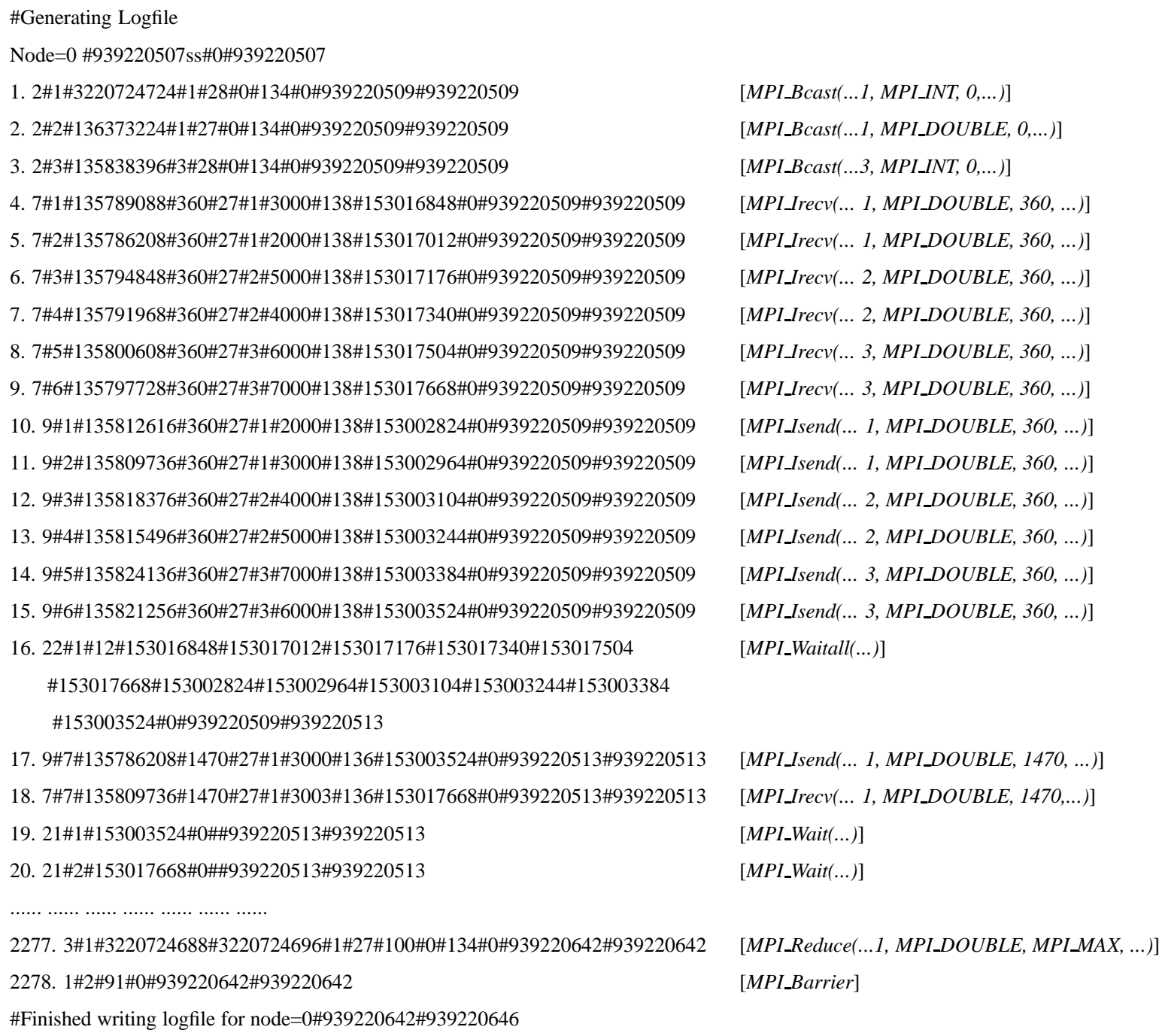

Data buffer pointer (3220724724)

Count of data elements (1)

Type of data elements ( 28 representing MPI_INT)

Node ID of root of broadcast tree ( 0 )

MPI Communicator ID (134)

Host ID (0)

Start Time of Call (939220509 msecs)

End Time of Call (939220509 msecs) 
Table 3 lists the raw trace, along with summarized call information that includes the type of the call, destination node number, data type, and message size, wherever applicable.

The next phase of the skeleton construction procedure consists of assigning symbols to identical or similar communication events. For this example, it was sufficient to keep the similarity threshold, discussed in section 4.2, at 0 to generate an acceptable trace. Hence every distinct MPI event is assigned a different symbol. Table 4 lists the symbols representing communication calls and the corresponding MPI events.

Table 4: Symbol table for MPI events

\begin{tabular}{|c|c|}
\hline P1: MPI_Irecv( $\ldots$ 1, MPI_DOUBLE, $360, \ldots)$ & P2: MPI_Irecv( $\ldots$ 1, MPI_DOUBLE, $1470, \ldots)$ \\
\hline P3: MPI_Irecv(... 1, MPI_DOUBLE, $245, \ldots)$ & P4: MPI_Irecv(... 3, MPI_DOUBLE, 360, ...) \\
\hline P5: MPI_Irecv(... 3, MPI_DOUBLE, 1470, ...) & P6: MPI_Irecv(... 3, MPI_DOUBLE, 245,...) \\
\hline P7: MPI_Irecv( $\ldots$ 2, MPI_DOUBLE, $360, \ldots)$ & P8: MPI_Irecv(... 2, MPI_DOUBLE, 1470, ...) \\
\hline P9: MPI_Irecv(... 2, MPI_DOUBLE, 245,...) & P10: MPI_Isend(... 1, MPI_DOUBLE, 360, ...) \\
\hline P11: MPI_Isend(... 1, MPI_DOUBLE, $1470, \ldots)$ & P12: MPI_Isend(... 1, MPI_DOUBLE, 245,...) \\
\hline P13: MPI_Isend(... 3, MPI_DOUBLE, 360, ...) & P14: MPI_Isend(... 3, MPI_DOUBLE, 1470, ...) \\
\hline P15: MPI_Isend(... 3, MPI_DOUBLE, 245,...) & P16: MPI_Isend(... 2, MPI_DOUBLE, 360, ...) \\
\hline P17: MPI_Isend(... 2, MPI_DOUBLE, $1470, \ldots)$ & P18: MPI_Isend(... 2, MPI_DOUBLE, 245,...) \\
\hline M1: MPI_Allreduce(... 5, MPI_DOUBLE, MPI_SUM, ...) & M2: MPI_Barrier \\
\hline M3: MPI_Reduce(...1, MPI_DOUBLE, MPI_MAX, ...) & M4: MPI_Bcast( $(. .1$, MPI_INT, $0, \ldots)$ \\
\hline M5: MPI_Bcast(...1, MPI_DOUBLE, $0, \ldots)$ & M6: MPI_Bcast(...3, MPI_INT, $0, \ldots)$ \\
\hline O1: MPI_Wait(...) & O2: MPI_Waitall(...) \\
\hline
\end{tabular}

After the clustering phase, which consists of replacing the MPI events with symbols according to the listing in Table 4 in this example, the part of the trace shown in Table 3 will be represented by the following string:

\{M4,M5,M6,P1,P1,P7,P7,P4,P4,P10,P10,P16,P16,P13,P13,O2,P11,P2,O1,O1,.........,M3,M2\}

The next step is to identify the cycles in this trace by applying the algorithm in Table 1. For this example we find that there is a single large loop that iterates 60 times. The final execution signature is represented as follows:

\{M4, M5, M6, L_0, M2, (L_0:60), M1, (P1:2), (P7:2), (P4:2), (P10:2), (P16:2), (P13:2), O2, M1, M3, M2\}

The format Symbol:Number implies that Symbol is repeated Number times. In the above signature, all symbols represent MPI calls as listed in Table 4, except that L_0 represents a loop consisting of 37 MPI calls listed as follows:

L_0 = [(P1:2), (P7:2), (P4:2), (P10:2), (P16:2), (P13:2), O2, P11, P2, (O1:2), P12, P3, (O1,2), P17, P8, 
(O1:2), P18, P9, (O1:2), P14, P5, (O1:2), P15, P6, (O1:2)]

The final step is to generate a specific performance skeleton program based on the scaling factor discussed in section 4.3. For this example, the skeleton always consists of the code representing Loop L_0 described above, while the number of iterations of the loop in the skeleton is determined by the scaling factor. Since the number of trace events outside of this loop is very small as compared to the events represented in the loop, the skeleton can be limited to this loop. The pseudo code for the skeleton is shown in Table 5 .

Table 5: Example skeleton code

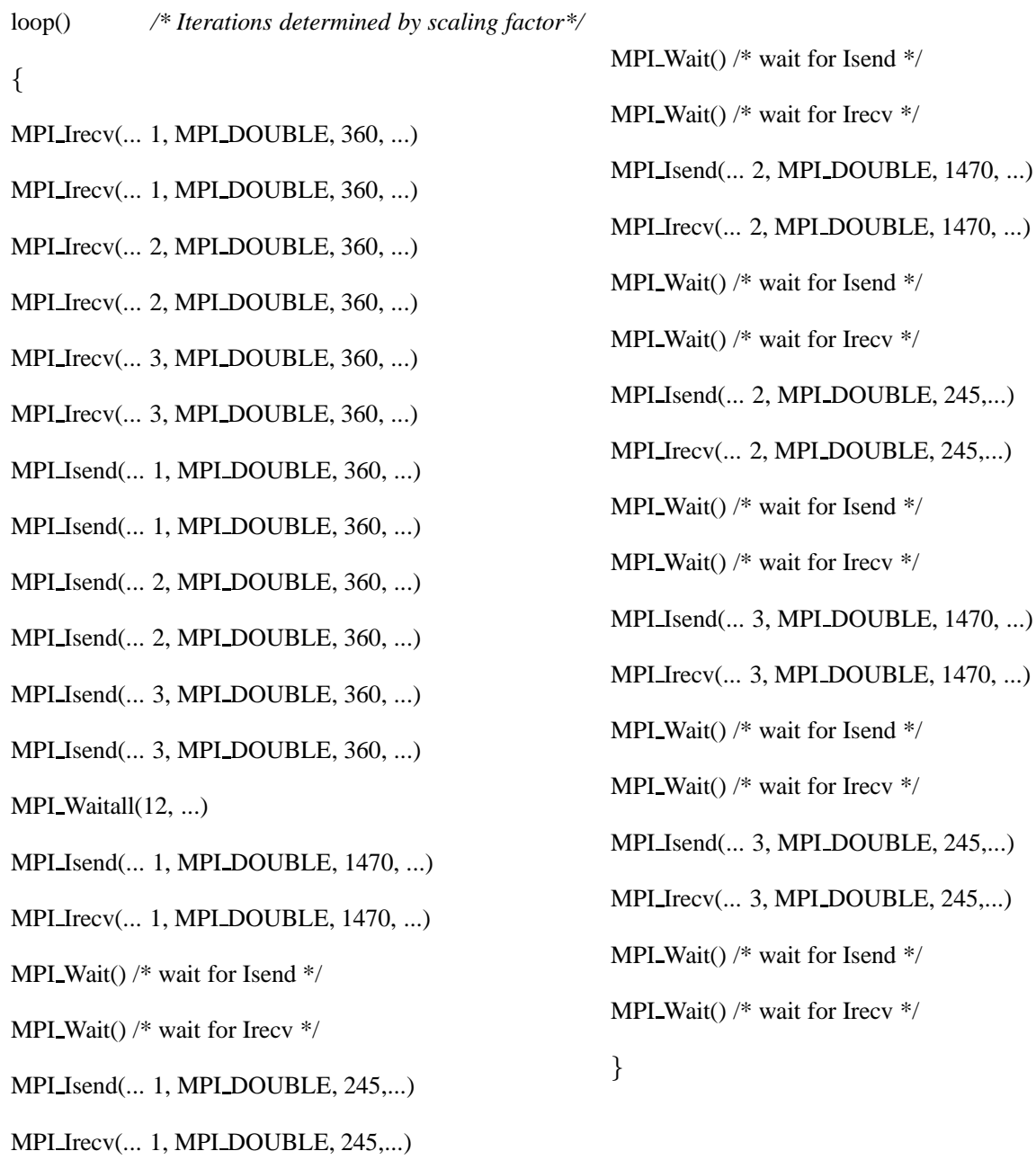




\subsection{Shortest running "good" skeleton}

It is desirable that the performance skeletons be short running since the execution of the performance skeleton is an overhead in performance estimation. However, the prediction accuracy is likely to be lower for shorter running skeletons. The framework we have developed is designed to construct skeletons for any scaling factor that is provided, and equivalently, for an arbitrary skeleton execution time. A key question in this research is as follows: How short running can a skeleton be and still generate reasonable performance estimates?

To address this, the skeleton construction framework heuristically determines the shortest runtime skeleton that it believes can be constructed without significantly sacrificing prediction accuracy, and issues a warning if the requested scaling factor implies a smaller skeleton. To determine the shortest "good" skeleton, the framework identifies the dominant sequence of execution events in the application that comprise a significantly large percentage of application execution time. A skeleton is considered a good skeleton if at least one full iteration of the dominant sequence of execution events is included.

As an example, consider the NAS IS (Integer Sort) benchmark whose main communication operation is a large all-to-all transfer. The accuracy of the skeleton is expected to be good if one or more full all-to-all transfers are included. Hence the minimum size for a good skeleton is the shortest skeleton that includes at least one full all-to-all transfer.

\section{Experiments and results}

A prototype framework for automatic construction of performance skeletons has been implemented. It was employed to generate skeletons to predict the performance of the corresponding applications on a network testbed.

\subsection{Experimental setup}

The testbed for the experiments is a compute cluster composed of 10 Intel Xeon dual CPU $1.7 \mathrm{GHz}$ machines connected by Gigabit Ethernet links and a full crossbar switch. Results are presented for experiments conducted on 4 nodes. All experimental results are based on the MPI implementation of the NAS Parallel Benchmarks [3, 32]. The codes used are BT (Block Tridiagonal solver), CG (Conjugate Gradient), IS (Integer Sort), LU (LU Solver), MG (Multigrid) and SP (Pentadiagonal solver). All programs are compiled using GNU g7 7 (Fortran) compiler except IS, which is compiled with the gcc (C) compiler. The MPICH implementation of MPI is used. The bandwidth between computation nodes was managed with the Linux advanced networking iproute 2 [2] in order to simulate limited bandwidth availability due to competing network traffic. iproute 2 works by intercepting the network packets and passing them through artificial queues to simulate 
bandwidth limitations.

Performance skeletons were constructed for each Class B NAS Benchmark program with an intended skeleton execution time of 10 seconds, 5 seconds, 2 seconds, 1 second and 0.5 second by defining the appropriate scaling factors.

\subsection{Validation of skeleton properties}

The performance skeletons are expected to have execution behavior that reflects the application. As a basic test, we compared the percentage of time spent in the communication (MPI) operations versus other computations for the skeletons and the application. The results are illustrated in Figure 2.

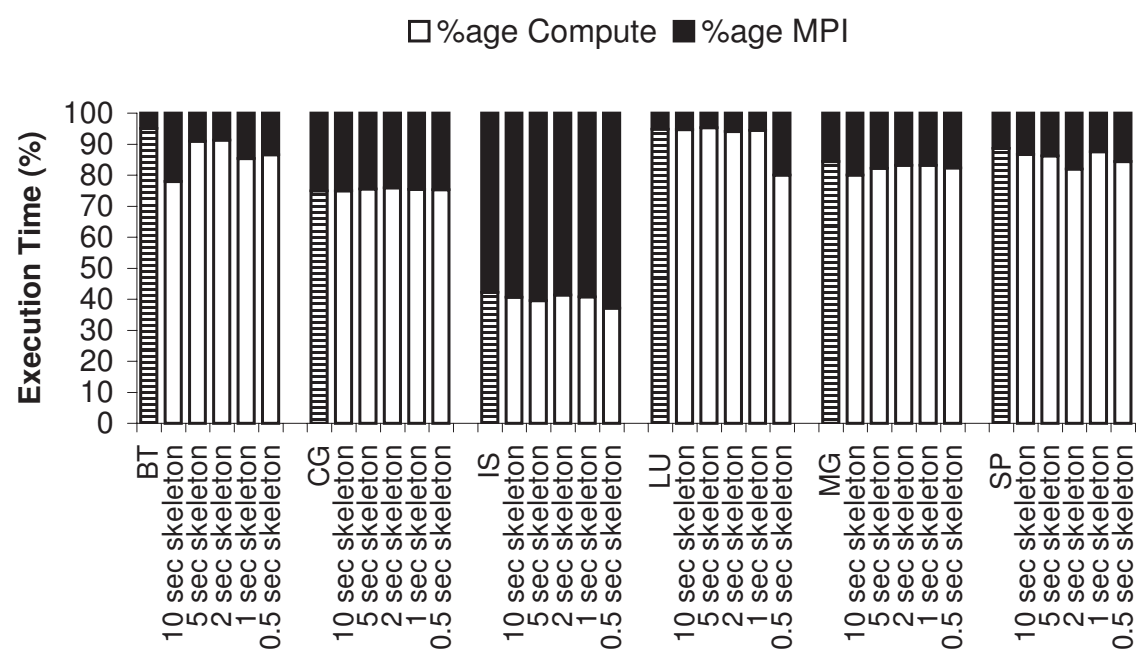

Figure 2: Time spent by NAS benchmarks and corresponding skeletons in different execution activities. The bar with horizontal lines is for the actual application.

We observe that the ratio between the computation and communication time is broadly similar for the skeletons and the corresponding application. The 0.5 second skeleton for the LU benchmark shows a somewhat larger communication time ratio than the other cases. We expect that very small skeletons will not represent the application as faithfully as larger skeletons as more approximations are involved in their construction. The ratios for the skeletons of BT benchmark show more variation than others. The conclusion is that moderate variations are possible because of the nature of skeleton construction process but most skeletons are fairly close to their application in this respect. 


\subsection{Validation of performance prediction}

In order to validate the ability of performance skeletons to predict application performance in different scenarios the NAS benchmarks and the corresponding performance skeletons were executed on the same testbed under the following five resource sharing scenarios:

1. Two competing compute intensive processes are run on one node.

2. Two competing compute intensive processes are run on each node.

3. Available bandwidth on one of the links was artificially limited to $10 \mathrm{Mbps}$.

4. Available bandwidth on each link was artificially limited to 10Mbps.

5. Competing processes as above on one node and reduced bandwidth as above on one link.

(Note that at least two competing processes are required to create meaningful CPU contention for an application process on dual processor nodes.)

We define the measured scaling ratio as the ratio between the measured execution time of an application and the measured execution time of a corresponding skeleton. The predicted execution time of an application in a resource sharing scenario is the product of the measured skeleton execution time in the same scenario and the corresponding measured scaling ratio. The predicted and measured application execution times were compared for different skeleton sizes and across different resource sharing scenarios. The remainder of this section discusses the results.

Average error in execution time predicted by the performance skeletons across applications and skeleton sizes is plotted in Figure 3. These results are averaged across resource sharing scenarios. We observe that the average prediction error across all benchmarks, scenarios and skeleton sizes is a relatively low $6.7 \%$ implying that the performance skeletons can predict execution time effectively. We now discuss the relationship of prediction accuracy to application characteristics, skeleton size, and resource sharing scenarios.

\section{Skeleton size and benchmarks:}

Our goal of "short running" performance skeletons is to reduce overheads but preserve prediction accuracy. From Figure 3 we observe that the relationship between average prediction error and skeleton size shows no distinct pattern across benchmarks. For some benchmarks, prediction error does not change much when going from 10 second to 0.5 second skeletons. However, error is usually close to the highest for the smallest 0.5 second skeletons. The average error across all applications for 0.5 seconds skeletons is around $8 \%$ versus the range around $5 \%$ to $6 \%$ for other cases. 


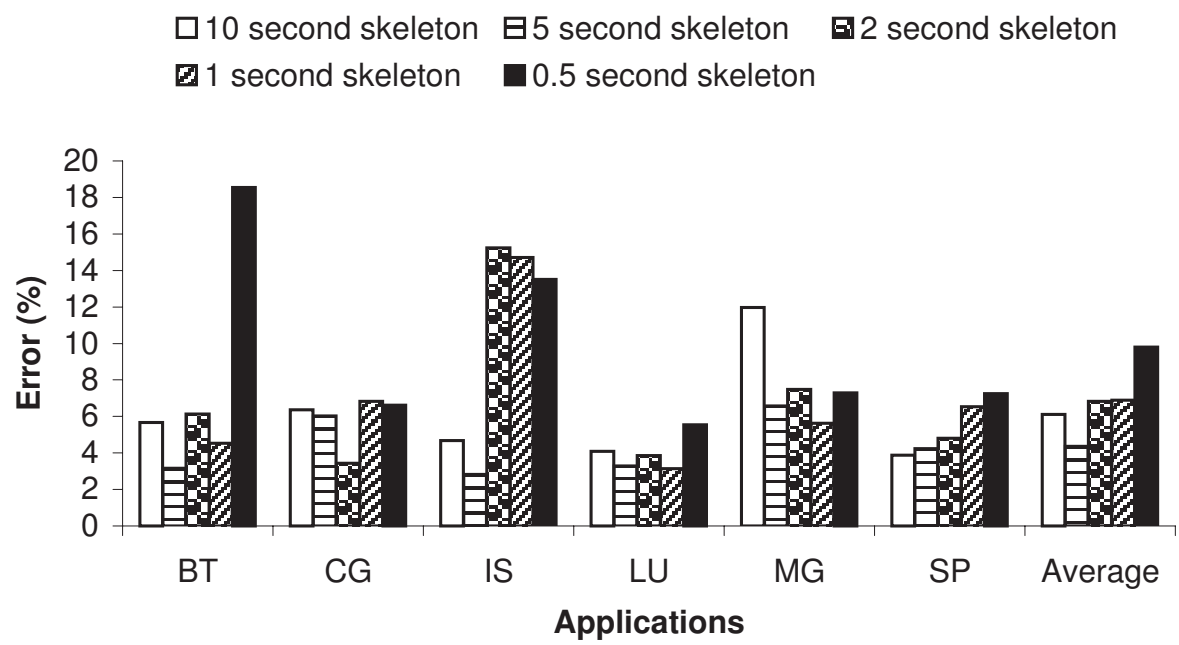

Figure 3: Prediction error for NAS benchmarks across skeletons sizes from 10 to 0.5 seconds. The error is averaged across all resource sharing scenarios.

The minimum execution time of a "good" skeleton for each benchmark as determined by our framework, based on discussion in section 4.5, is listed in Figure 4. Based on this table, the skeletons that are flagged as potentially "not good" are 0.5 and 1 second skeletons for BT, $0.5,1$, and 2 second skeletons for IS, and 0.5 and 1 second skeletons for LU. Indeed the 4 cases with the highest prediction error, i.e., the 0.5 second BT skeleton and $0.5,1$, and 2 second IS skeletons, were flagged to have low prediction value by the skeleton construction framework.

\begin{tabular}{|c|c|}
\hline Application & Smallest Skeleton \\
\hline BT & $1.01 \mathrm{sec}$ \\
\hline CG & $0.13 \mathrm{sec}$ \\
\hline IS & $3 \mathrm{sec}$ \\
\hline LU & $1.97 \mathrm{sec}$ \\
\hline MG & $0.34 \mathrm{sec}$ \\
\hline SP & $0.34 \mathrm{sec}$ \\
\hline
\end{tabular}

Figure 4: Estimated minimum execution time for the smallest "good" skeleton.

The prediction errors for each skeleton size are grouped together and displayed in Figure 5. While there is no uniform pattern again, the number of cases with a relatively large prediction error increase with decreasing skeleton size and is clearly the highest for 0.5 second skeletons.

The main conclusion is that performance skeletons of a few seconds are normally adequate for reasonably 


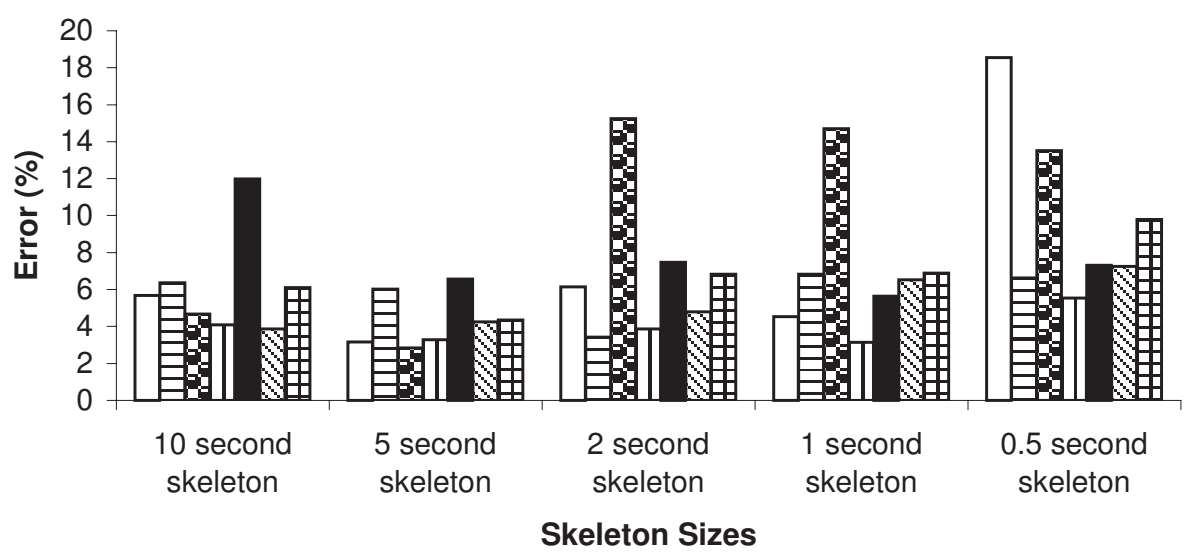

Figure 5: Prediction error for skeletons of different sizes for NAS benchmarks. The error is averaged across all resource sharing scenarios.

accurate performance prediction, with a loose correlation between smaller skeletons and lower prediction accuracy. Also, the framework generates meaningful application specific lower bounds for skeleton sizes.

\section{Sharing scenarios:}

We examine how the nature of sharing relates to accuracy of performance prediction. Our experiments have spanned sharing of one or all CPUs, one or all communication links, and a combination of one node and one link. Figure 6 shows prediction error under different sharing scenarios when employing representative 10 second skeletons. We observe that the prediction error is higher for scenarios that include competing traffic. In the case of CPU sharing only, the error is higher for the "unbalanced" sharing of a single node versus sharing of all nodes.

We believe that prediction error is higher for network sharing because communication operations cannot be scaled down linearly unlike compute operations, as discussed in section 4.3. We speculate that the error in unbalanced execution scenarios is higher because of potential inaccuracy in reproduction of synchronization behavior in performance skeletons. While constructing a skeleton, we set the duration of compute operations within loops to their average duration across iterations of the loop. A more accurate approach that considers frequency distribution of the duration of compute events will be taken in the future. 


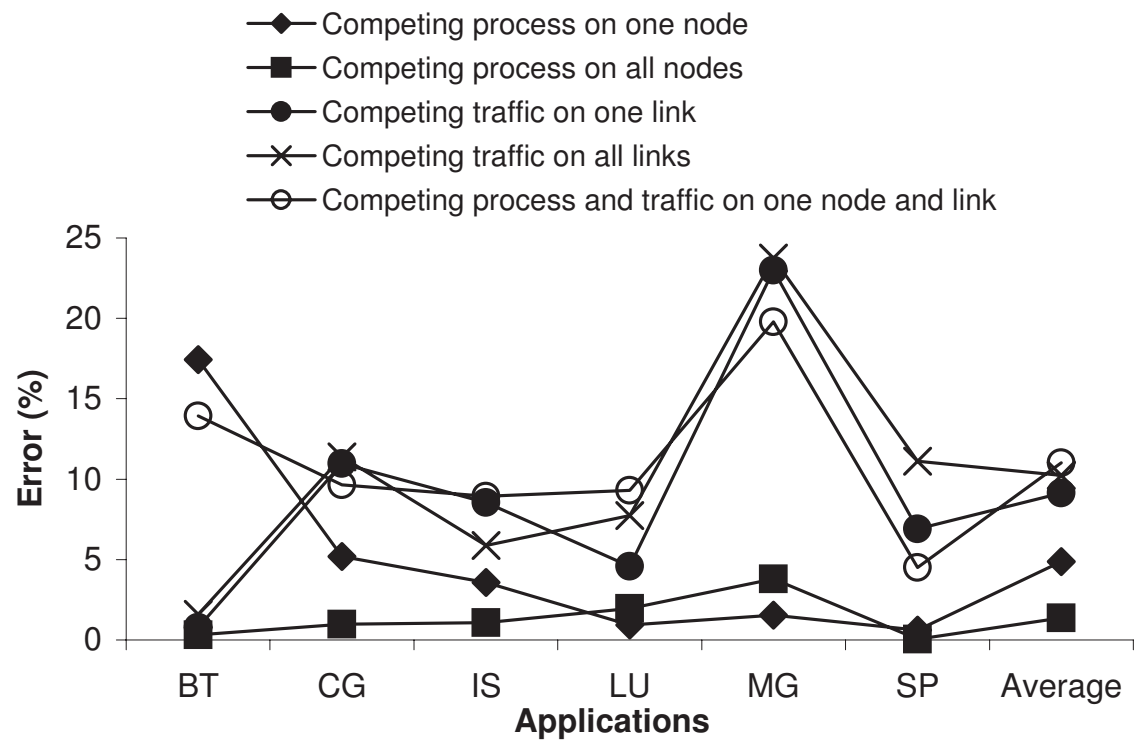

Figure 6: Prediction error for NAS benchmarks across five resource sharing scenarios. A 10 second skeleton was used.

\subsection{Comparison with other prediction techniques}

We performed additional experiments to compare the prediction accuracy of performance skeletons versus two other simple and "reasonable" approaches to performance prediction listed as follows:

Average Prediction: The average slowdown of the entire benchmark suite under a given resource sharing scenario was used to predict the execution time for every program in the same scenario. The reasoning is that, if all programs slow down roughly equally under resource competition, there is no need for customized performance skeletons for applications discussed in this paper; instead, a generic short running program could be employed to predict the execution time for any application under resource sharing.

Class S Prediction: The experiments described in this paper were performed with Class B NAS benchmarks, which run in 30 to 900 seconds without load on 4 machines in our cluster. Each NAS benchmark also has a Class $\mathrm{S}$ version which typically runs in less than a second. In this case, the Class $\mathrm{S}$ benchmarks were used as the performance skeletons for the Class B benchmarks for performance prediction. The reasoning is that, since both classes of benchmarks perform the same fundamental calculation but on different data sizes and scales, the short running class $\mathrm{S}$ benchmarks could be considered good manually generated performance skeletons. 


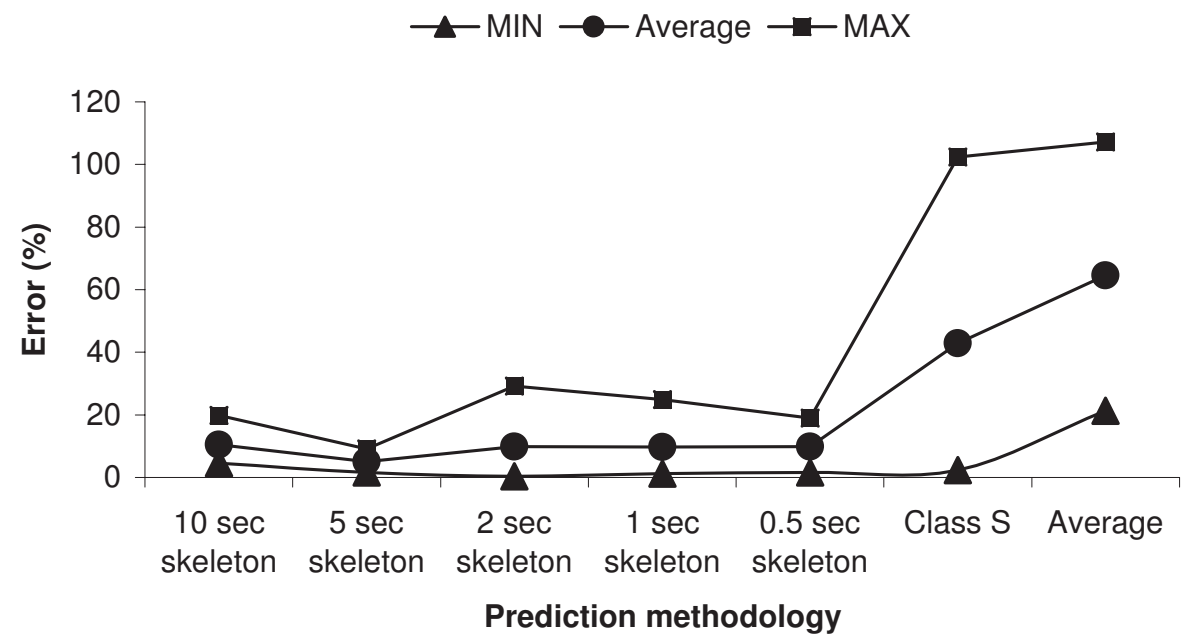

Figure 7: Minimum, maximum and average prediction error for the NAS benchmark suite for prediction with different size skeletons, with class $\mathrm{S}$ benchmarks as skeletons, and using average prediction. The execution scenario is one competing process on one node and traffic on one link

The performance prediction error for each of these approaches is plotted in Figure 7. The performance skeleton approach based on the framework in this paper is clearly better than the other methods. Prediction with 0.5 second skeletons, which roughly take as long to run as Class $\mathrm{S}$ benchmarks, is also clearly superior to other methods. Hence the overhead of our approach is also competitive.

The above results are significant for the following reasons. The fact that "average prediction" approach is relatively ineffective proves that applications have widely varying execution behavior and hence an approach that is customized to applications is required. The inability of Class $\mathrm{S}$ benchmarks to predict the behavior of larger Class B benchmarks shows that one cannot simply run an application with a very small input data set and expect it to have similar execution behavior as running with realistic data sets.

\section{Limitations and extensions}

This research establishes performance skeletons as an important and effective approach to execution driven performance prediction. We discuss the limitations of our implementation and future work that is needed to develop a comprehensive solution to the problems this framework is designed to solve.

- Coordinated skeletons: In the current framework, skeletons are constructed independently for each executing process and heuristics are employed to handle any communication mismatches among the skeleton processes. Ongoing research is addressing construction of coordinated skeletons where a 
single SPMD skeleton program is constructed by employing a global analysis of all process traces.

- Scalable skeletons: In the current methodology, the number of threads in a skeleton is the number of nodes employed in skeleton generation. Hence, a skeleton is constructed for execution on a fixed number of nodes (or threads) and the construction procedure would have to be repeated for a different number of nodes. Of course, it is desirable to have skeletons that can execute on, and provide a performance estimate for, any number of nodes. This is a challenge since the program execution behavior itself can change as the number of execution threads and nodes change. Our current research is investigating construction of parameterized skeletons by analyzing the execution behavior for a range of node sets and employing the coordinated skeleton approach discussed above.

- Efficiency of skeleton construction: The execution time of skeleton construction is dominated by the compression procedure that employs an $O\left(N^{3}\right)$ algorithm, as noted in section 4.2. Tradeoffs between the degree of compression and compression time are possible and may be necessary for long running programs with frequent communication calls. In particular, if the longest repeating pattern is limited to length $L$, the complexity can be trivially reduced to $O\left(N^{2} L\right)$. Ongoing work is exploring more efficient algorithms and tradeoffs.

- Fine grain prediction: This paper is limited to modeling coarse computation and communication behavior. Modeling of instruction level execution and memory access patterns is essential to employ skeletons to predict performance across architectures. An important application is prediction of performance by simulation on a future architecture.

- Other programming models: The current implementation is limited to message passing MPI programs. While the basic concepts are independent of the programming model, implementation will be significantly different for another programming model. An alternate approach that infers communication by network modeling can be independent of the programming model and has been investigated in $[26]$.

- Prediction across data sets: The current methodology constructs a skeleton based on a specific execution and input data set. The approach can be made to work across different data set sizes when execution time is dependent on the size of a data set. However, like most performance prediction methods, this approach cannot be applied when execution is strongly data dependent.

- Implementation and experimentation: Several aspects of this implementation can be improved. Synchronization overhead is modeled in a simple way as the timing information is approximated. Scaling down of individual communication operations can be more sophisticated. More experimentation, particularly on wide area networks, is needed for stronger validation. 


\section{Conclusion}

This paper introduces performance skeletons, which are short running programs that are constructed to mimic an application. Monitored skeleton execution in any runtime environment yields an estimate of the performance of the corresponding application in that environment. The key advantage of this approach is that modeling of the runtime environment is not required. Hence, it is particularly suitable for execution in dynamic and unpredictable environments. Our driving scenario is a grid environment, where modeling the dynamic network and host availability can be difficult to impossible.

The paper details how performance skeletons can be generated automatically from execution traces on an ordinary cluster. We demonstrate that automatically generated performance skeletons that run in seconds can predict application performance accurately. The average prediction error for 5 and 10 second skeletons for a variety of scenarios involving network and node sharing was 5-6\%. Our framework can also effectively compute the size of the shortest possible "good" skeleton that can estimate performance accurately. The results show that good skeletons executing in 2 seconds or less can be constructed for all NAS benchmarks. The paper offers detailed results and insight into how application characteristics, skeleton size and nature of resource competition impact prediction accuracy.

The paper discusses the limitations of this approach in detail and points out the major directions in which improvements are important. One of the goals is to instigate more research into this approach to performance prediction. In summary, the paper presents a promising approach to performance estimation with resource sharing and provides convincing evidence that it is practical and effective.

\section{Acknowledgments}

This material is based upon work supported by the National Science Foundation under Grant No. ACI0234328 and Grant No. CNS-0410797. Support was also provided by the Department of Energy through Los Alamos National Laboratory (LANL) contract number 03891-99-23, and by University of Houston's Texas Learning and Computation Center.

\section{References}

[1] Linux man pages.

[2] W. Almesberger. Linux network traffic control — implementation overview. White Paper, April 1999. Available at ftp://lrcftp.epfl.ch/pub/people/almesber/pub/tcio-current.ps. 
[3] D. Bailey, T. Harris, W. Saphir, R. van der Wijngaart, A. Woo, and M. Yarrow. The NAS Parallel Benchmarks 2.0. Technical Report 95-020, NASA Ames Research Center, December 1995.

[4] F. Berman, R. Wolski, S. Figueira, J. Schopf, and G. Shao. Application-level scheduling on distributed heterogeneous networks. In Proceedings of Supercomputing '96, Pittsburgh, PA, November 1996.

[5] J. Bolliger and T. Gross. A framework-based approach to the development of network-aware applications. IEEE Trans. Softw. Eng., 24(5):376 - 390, May 1998.

[6] R. Buyya, D. Abramson, and J. Giddy. Nimrod/G: An architecture for a resource management and scheduling system in a global computational grid. In The 4th International Conference on High Performance Computing in Asia-Pacific Region, 2000.

[7] N. Cardwell, S. Savage, and T. Anderson. Modeling TCP latency. In Proceedings of IEEE INFOCOM 2000 , pages $1742-1751,2000$.

[8] H. Casanova and J. Dongarra. NetSolve: A network-enabled server for solving computational science problems. The International Journal of Supercomputer Applications and High Performance Computing, 11(3):212-223, Fall 1997.

[9] H. Casanova, G. Obertelli, F. Berman, and R. Wolski. The AppLeS Parameter Sweep Template: Userlevel middleware for the grid. In Supercomputing 2000, pages 75-76, 2000.

[10] M. Dikaiakos, A. Rogers, and K. Steiglitz. Fast: A functional algorithm simulation testbed. In International Conference On Parallel and Distributed Systems, December 1993.

[11] P. Dinda and D. O'Hallaron. An evaluation of linear models for host load prediction. In Proceedings of the 8th IEEE International Symposium on High Performance Distributed Computing, August 1999.

[12] E. Duesterwald, C. Cascaval, and S. Dwarkadas. Characterizing and predicting program behavior and its variability. In International Conference on Parallel Architectures and Compilation Techniques (PACT), New Orleans, LA, September 2003.

[13] J. Han and M. Kamber. Data Mining: Concepts and techniques. Morgan Kaufman Publishers, 2001.

[14] K. Lai and M. Baker. Nettimer: A tool for measuring bottleneck link bandwidth. In USENIX Synposium on Internet Topology and Systems, pages 123-134, March 1991.

[15] F. M. Leo T. Yang, Xiaosong Ma. Cross-platform performance prediction of parallel applications using partial execution. In Proceedings of Supercomputing '05, Seattle, WA, Nov 2005. 
[16] M. Litzkow, M. Livny, and M. Mutka. Condor - A hunter of idle workstations. In Proceedings of the Eighth Conference on Distributed Computing Systems, San Jose, California, June 1988.

[17] B. Lowekamp, N. Miller, D. Sutherland, T. Gross, P. Steenkiste, and J. Subhlok. A resource query interface for network-aware applications. In Seventh IEEE Symposium on High-Performance Distributed Computing, Chicago, IL, July 1998.

[18] C. Lu and D. A. Reed. Compact application signatures for parallel and distributed scientific codes. In Proceedings of Supercomputing 2002, Baltimore,MD, Nov 2002.

[19] Message Passing Interface Forum. MPI: A message-passing interface standard. Technical Report UTCS-94-230, 1994.

[20] M. Nakazawa, D. K. Lowenthal, and W. Zhou. The MHETA execution model for heterogeneous clusters. In Proceedings of Supercomputing '05, Seattle, WA, Nov 2005.

[21] V. Paxson and S. Floyd. Wide-area traffic: The failure of Poisson modeling. IEEE/ACM Transactions on Networking, 3(3):226-244, June 1995.

[22] R. Raman, M. Livny, and M. Solomon. Matchmaking: Distributed resource management for high throughput computing. In 7th IEEE International Symposium on High Performance Distributed Computing, July 1998.

[23] G. Shao, F. Berman, and R. Wolski. Master/slave computing on the grid. In 9th Heterogeneous Computing Workshop, pages 3-16, 2000.

[24] T. Sherwood, E. Perelman, and B. Calder. Basic block-dsitribution analysis to find periodic behavior and simulation points in applications. In International Conference on Parallel Architectures and Compilation Techniques (PACT), Sep 2001.

[25] T. Sherwood, E. Perelman, G. Hamerly, and B. Calder. Automatically characterizing large scale program behavior. In 10th International Conference on Architectural Support for Programming Languages and Operating Systems (ASPLOS-X), San Jose, CA, October 2002.

[26] A. Singh and J. Subhlok. Reconstruction of application layer message sequences by network monitoring. In IASTED International Conference on Communications and Computer Networks, Boston, MA, November 2002.

[27] A. Snavely, L. Carrington, N. Wolter, J. Labarta, R. Badia, and A. Purkayastha. A framework for performance modeling and prediction. In Proceedings of Supercomputing 2002, Baltimore,MD, Nov 2002. 
[28] A. Snavely, N. Wolter, and L. Carrington. Modeling application performance by convolving machine signatures with application profiles. In IEEE Workshop on Workload Characterization, Austin, TX, 2001.

[29] S. Sodhi. Automatic construction of performance skeletons for grid resource selection and performance estimation frameworks. Master's thesis, University of Houston, Jan 2004.

[30] M. Stemm, S. Seshan, and R. Katz. Spand: Shared passive network performance discovery. In USENIX Symposium on Internet Technologies and Systems, Monterey, CA, June 1997.

[31] J. Subhlok, P. Lieu, and B. Lowekamp. Automatic node selection for high performance applications on networks. In Proceedings of the Seventh ACM SIGPLAN Symposium on Principles and Practice of Parallel Programming, pages 163-172, Atlanta, GA, May 1999.

[32] T. Tabe and Q. Stout. The use of the MPI communication library in the NAS Parallel Benchmark. Technical Report CSE-TR-386-99, Department of Computer Science, University of Michigan, Nov 1999.

[33] H. Tangmunarunkit and P. Steenkiste. Network-aware distributed computing: A case study. In Second Workshop on Runtime Systems for Parallel Programming (RTSPP), Orlando, March 1998.

[34] A. Toomula and J. Subhlok. Replication memory behavior for performance prediction. In LCR 2004: The 7th Workshop on Languages, Compilers, and Run-time Supportfor Scalable Systems, Houston, TX, October 2004.

[35] S. Venkataramaiah and J. Subhlok. Performance estimation for scheduling on shared networks. In 9th Workshop on Job Scheduling Strategies for Parallel Processing, Seattle, WA, June 2003.

[36] J. Weismann. Metascheduling: A scheduling model for metacomputing systems. In Seventh IEEE Symposium on High-Performance Distributed Computing, Chicago, IL, July 1998.

[37] R. Wolski, N. Spring, and J. Hayes. Predicting the CPU availability of time-shared unix systems on the computational grid. Cluster Computing, 3(4):293-301, 2000.

[38] R. Wolski, N. Spring, and C. Peterson. Implementing a performance forecasting system for metacomputing: The Network Weather Service. In Proceedings of Supercomputing '97, San Jose, CA, Nov 1997. 